\title{
La ansiedad aumenta el riesgo de muerte por enfermedad isquémica cardíaca
}

\author{
Psychological characteristics and fatal ischaemic heart disease. Haines A, Cooper J, Meade TW. Heart. $2001 ; 85$ :385-389.
}

\section{Objetivo}

Determinar la relación entre características psicológicas y subsecuentes eventos fatales de enfermedad isquémica cardíaca.

\section{Diseño}

Estudio de cohorte prospectivo.

Lugar

Noroeste de Londres. Gran Bretaña.

\section{Participantes}

1408 hombres blancos entre 40 y 64 años, sin historia de infarto de miocardio previa, que completaron el Ínventario de Crown-Crisp. Fueron reclutados entre los años 1972 y 1978 y con seguimiento de casos fatales de enfermedad cardíaca isquémica hasta 1997.

\section{Evaluación de factores de riesgo}

Los participantes completaron un inventario de 48 preguntas con puntuaciones de 0 a 16 para cada una. Éstas se encuentran agrupadas en seis escalas que evalúan respectivamente: ansiedad flotante, ansiedad fóbica, obsesividad/neurosis obsesiva, quejas somáticas funcionales, depresión e histeria (extroversión). Se evaluaron también los factores de riesgo cardiovasculares habituales.

\section{Medición de resultados principales}

Se notificaron todos los casos de muerte registrados por el Servi- cio Nacional de Salud entre abril de 1972 y julio de 1997. Se adjuntó información acerca de la historia clínica del paciente, resultado de estudios diagnósticos realizados incluyendo ECG y enzimas cardíacas, así como los resultados de exámenes postmortem. La información obtenida para determinar si la causa de muerte se debió a enfermedad cardíaca isquémica (criterios de la OMS) fue realizada por tres profesionales ciegos a los factores de riesgo de los pacientes.

\section{Resultados principales}

Durante el período de seguimiento, 127 de los participantes fallecieron por enfermedad cardíaca isquémica Cada punto de incremento en la escala de obsesividad/neurosis obsesiva se asoció con un riego relativo de sufrir un episodio fatal de enfermedad cardíaca isquémica de 1.08 (IC 95\% 1.02 a 1.15). Para la escala de quejas somáticas funcionales el riesgo relativo fue también de 1.08 (IC $95 \% 1.02$ a1.15). Respecto a la puntuación total, es decir la del conjunto de las seis escalas, el riesgo relativo fue de 1.28 (IC 95\% 1.09 a 1.50). La asociación fue independiente de la edad, clase social y factores de riesgo conocidos para enfermedad cardiovascular.

\section{Conclusion}

Tanto el puntaje total del Inventario, como los de obsesividad y de quejas somáticas funcionales se asociaron a episodios fatales de enfermedad cardíaca isquémica a largo plazo, independientemente de otros factores de riesgo conocidos.

Fuente de financiamiento: no referida

\section{Comentario}

En este estudio se intenta establecer la asociación entre características psicológicas que conformarían diversas manifestaciones de ansiedad y el riesgo de muerte por enfermedad cardíaca isquémica. Se utilizó para tal fin un inventario con seis escalas, que evalúan diferentes agrupaciones sintomáticas a fin verificar la presencia, intensidad y caracterización de síntomas de ansiedad de los participantes y el subsecuente riesgo de muerte por cardiopatía isquémica.

Se estableció una asociación significativa con el incremento de puntaje en dos de las escalas (obsesividad/neurosis obsesiva y quejas somáticas funcionales), así como con el puntaje total. Es de destacar que los autores habían hallado una asociación significativa con ansiedad fóbica en un estudio anterior, siendo esta evidente sólo en los primeros diez años del seguimiento y debilitándose luego. Ellos lo atribuyen a la variación que los síntomas fóbicos tendrían a lo largo del tiempo en comparación a los síntomas evaluados por la escala de obsesividad/neurosis obsesiva que incluiría ítems que darían cuenta de características de personalidad, más estabies en el tiempo. Es decir que la persistencia sostenida de los síntomas parecería tener importancia. Los mecanismos de la asociación establecida están sujetos a debate, postulando que la ansiedad podría incrementar el riego de muerte por cardiopatía isquémica al influenciar conductas (por ej., fumar), promoviendo la aterogénesis o desencadenando eventos coronarios fatales. Los autores encuentran más compatible la tercera de las hipótesis. La ansiedad podría asociarse con anomalías en el control autonómico que podrían incrementar el riesgo de arritmias ventriculares fatales ${ }^{1}$ o en la vasoconstricción de segmentos ateroescleróticos de arterias coronarias frente a eventos productores de ansiedad. 2 De ser así, la posibilidad de controlar la ansiedad, permitiría disminuir el riesgo de muerte por cardiopatía isquémica. Quisiera hacer un breve comentario respecto a la agrupación de síntomas de las escalas, y a la posibilidad de identificar entidades que permitan ser tratadas adecuadamente a fin de reducir el riesgo men- cionado. Me refiero específicamente a la escala de obsesividad/neurosis obsesiva, ya que los autores sugieren evaluar el impacto que la terapia cognitivo conductual tendría en la reducción del riesgo en pacientes con puntuación elevada. Ellos dan por sentado la efectividad de ésta en el tratamiento de dichos síntomas. En realidad, la misma sólo ha demostrado su efectividad en pacientes que sufren un trastorno obsesivo compulsivo (DSM-IV). También la utilización de antidepresivos inhibidores de la recaptación de serotonina (clomipramina, fluoxetina, fluvoxamina, etc.) ha sido demostrada como eficaz en dicha patología. Sin embargo, no puede afirmarse hoy lo mismo respecto a la efectividad del tratamiento para pacientes que presentan un trastorno de personalidad obsesivo compulsivo (eje II del DSMIV) y no un trastorno obsesivo compulsivo (eje I del DSM-IV). ${ }^{3}$ Probablemente, entre los participantes con alta puntuación en la escala de obsesividad/neurosis obsesiva nos encontremos con cierta heterogeneidad diagnóstica dado el tipo de escala. Algunos, a quienes pueda corresponder el diagnóstico de trastorno obsesivo compulsivo (DSM-IV), muchos otros a los que debería diagnosticarse como trastorno de la personalidad de tipo obsesivo compulsivo (DSM-IV) y algunos con una combinación de ambos. Si así fuera, solo podría haber evidencia suficiente en la efectividad de la terapia cognitivo conductual, así como en la indicación de antidepresivos inhibidores de la recaptación de serotonina en aquellos pacientes que padecen un trastorno obsesivo compulsivo y no en los que presentaran personalidad obsesivo compulsiva. El abordaje de este último grupo debería realizarse con otros enfoques psicoterapéuticos, con eficacia a ser demostrada.

Los alcances de este estudio sugieren que padecer trastornos de ansiedad es un factor de riesgo independiente de mortalidad por cardiopatía isquémica. Lo que no permite afirmar es que el tratamiento de los trastornos de la ansiedad reduzcan el riesgo de muerte por cardiopatía isquémica.

\section{Dr. Fabian J. Triskier [ Servicio de Psiquiatría. Hospital P.Piñero ]}

\section{Referencias}

1. Kubzan .

3. Jenike, MA, Psychotherpy of Obsessive Compulsive Personality Disorder enJenike,MA, Baer L, Minichiello, WE, Obsessive- Compulsive Disorders. Practical Management, 1998 Mosby Inc. ; 611-624. 